\title{
Prendre soin des soignants: un programme innovant pour les infirmières en oncologie
}

\author{
Par Margaret I. Fitch, Yvette Matyas et Marny Robinette
}

\section{Résumé}

Un programme innovateur, Care for the Professional Caregiver [ci-après appelé Prendre soin des soignants professionnels], a été élaboré pour donner aux infirmières de chevet d'un programme de lutte contre le cancer l'occasion d'apprendre comment faire face au stress dans l'exercice de leur travail. Le programme a été évalué par des méthodes quantitatives et qualitatives. Les résultats démontrent clairement les bénéfices du programme tant à court terme qu'à long terme. Le fait de nouer des liens profonds avec d'autres infirmières en oncologie, de se soutenir mutuellement et d'échanger des récits sur leurs expériences au travail a été profitable aux infirmières sur les plans personnel, professionnel et collectif. Le programme a atteint ses objectifs, et on recommande la poursuite de sa mise en ouvre.

\section{Introduction et historique}

Malgré les avancées thérapeutiques et la baisse des taux de mortalité, un diagnostic de cancer est encore souvent interprété comme une sentence de mort (Loney, 1998). La plupart des malades pensent au mourir et à leur propre mort (Fitch et Steele, 2003). Ils éprouvent un sentiment d'impuissance et de perte de contrôle sur leur vie. Les 50 \% qui succombent à la maladie doivent composer avec le deuil anticipé et la planification des soins de fin de vie.

Les infirmières en oncologie qui soignent des personnes atteintes du cancer sont confrontées régulièrement à la mort et sont témoins des souffrances qu'elle occasionne. L'un des buts des soins infirmiers en oncologie est d'aider les personnes - les patients et leur famille - à faire face aux difficultés psychosociales relatives à leur expérience du cancer. Pour y parvenir, elles font une utilisation thérapeutique du soi et construisent des relations interpersonnelles avec les personnes concernées. Les soins infirmiers ont été décrits comme un « travail interpersonnel », permanent par nature, et incarné par une relation personnelle avec chaque patient dans un contexte organisationnel et social élargi (Corner, 2002). Les sentiments conscients et inconscients des infirmières s'inscrivent dans cette relation et entrent dans les échanges avec les patients (Lancely, 2001). En occupant une place dans le monde du patient mourant, les infirmières partagent le fardeau de ses souffrances grâce $\mathrm{au}$ « caring », à l'empathie et à la compassion (King et Jordan-Welch, 2003). Elles partagent également le chagrin et le deuil lorsque les patients décèdent (Feldstein et Gemma, 1995; Saunders et Valente, 1994).

Les soins infirmiers en oncologie sont reconnus depuis longtemps comme étant une spécialité difficile (Newlin et Wellisch, 1978; Wilkinson, 1994). Leurs exigences ont été décrites comme des sources de stress (Kushnir, Rabin et Azulai, 1997; Vachon, 1986), des facteurs d'épuisement (Lewis, 1999; McElroy, 1982; Yasko, 1983) et des difficultés liées à la pratique (Cohen et Sarter, 1992; Lev, 1994), illustrés par de nombreux exemples, tels le décès d'un patient préféré, les pertes multiples et la confrontation répétée avec la mort, les soins aux patients qui reçoivent des traitements désagréables, les conflits entre les médecins et les infirmières sur les buts des soins, la charge professionnelle élevée et les relations difficiles entre collègues de travail.

Durant la dernière décennie, au Canada, le réseau de prestation de soins aux personnes atteintes de cancer a connu des changements significatifs : réduction des moyens financiers, nouveaux modèles d'organisation, réforme de la santé, restructuration et fusionnements. La tendance à réduire les séjours à l'hôpital, en fournissant davantage de soins en milieu ambulatoire ou communautaire, ainsi que la plus grande complexité des protocoles de traitement du cancer ont accru la lourdeur et la complexité des cas. Les infirmières en cancérologie exposent les changements survenus dans leurs milieux de travail, la charge de travail, la structure hiérarchique, le nombre de collègues, les moyens disponibles et le leadership professionnel (Bakker, Fitch, Green, Butler et Olson, sous presse). Plusieurs d'entre elles avouent ressentir moins de satisfaction au travail et formulent des inquiétudes à propos de leur capacité à fournir des soins sûrs et des soins de qualité (Lamkin, Rosiak, Buerhaus, Mallory et Williams, 2002; Lovern, 2001). Ces variables organisationnelles sont présentées comme des sources majeures de stress pour les infirmières (Cohen, Haberman, Steeves et Deatrick, 1994; Corner, 2002).

Malgré tous ces changements, les infirmières en oncologie demeurent profondément dévouées envers leurs patients et fortement motivées à prodiguer de bons soins (Bakker et al., sous presse). Beaucoup s'efforcent de répondre aux besoins de leurs patients, quitte à payer de leur personne. Larson (1992) a décrit la lutte constante que constitue la recherche d'un moyen de s'investir émotionnellement auprès des patients mourants, tout en se préservant soi-même. Les infirmières ne peuvent pas véritablement accompagner et soutenir les patients jusqu'à la fin sans reconnaître au préalable leurs propres besoins et y répondre. Les métaphores employées par les infirmières pour décrire leur travail reprennent souvent les termes de bataille, de lutte et de combat (Cohen et Sarter, 1992; Kushnir, Rabin et Azulai, 1997). Beaucoup perçoivent les multiples défis qui se posent au quotidien dans le système actuel de lutte contre le cancer comme des entraves, les empêchant de « faire un véritable travail infirmier », et en retirent un sentiment de frustration et de désespoir (Bakker et al., sous presse).

Lorsque la détresse chez le soignant n'est pas maîtrisée, elle peut avoir un impact très négatif sur l'individu et sur les soins au patient. Dans la pratique de l'oncologie infirmière, un stress professionnel excessif et continu peut être associé à une variété d'émotions, telles l'anxiété et la dépression, l'insatisfaction au travail et une dégradation de la qualité de vie (Kushnir, Rabin et Azulai, 1997). Il a été établi que

Margaret I. Fitch, inf., PhD, est directrice de l'Unité de recherche psychosociale et comportementale, marg.fitch@sunnybrook.ca

Yvette Matyas, BA (Psych), M.Sc.inf., est directrice, Opérations.

Marny Robinette, BA, est gestionnaire de Wellspring; toutes trois cuvrent au Centre régional de cancérologie de TorontoSunnybrook, à Toronto, Ontario. 
le stress est impliqué dans de nombreuses maladies (par ex. les maladies cardio-vasculaires, le diabète) et de problèmes comportementaux au travail (par ex. l'absentéisme) (Lancely, 2001). Les conséquences possibles incluent de mauvaises performances au travail, le roulement important de la main-d'œuvre et l'abandon de la profession (Vachon, 1986).

Le défi qui se pose aux administrateurs et aux gestionnaires des programmes de lutte contre le cancer est de trouver les moyens de soutenir sérieusement et efficacement le personnel infirmier dans des milieux de pratique qui ne sont pas susceptibles d'évoluer à court terme. Les finances vont continuer à être restreintes, le nombre de patients par infirmière va continuer à être élevé, et les processus organisationnels vont continuer à être complexes. Les infirmières en oncologie doivent trouver des manières d'équilibrer leur vie professionnelle et leur vie privée, et des moyens de faire face aux exigences continuelles liées à la pratique de leur spécialité. Cet exposé décrit l'approche qu'un programme de lutte contre le cancer a adoptée pour soutenir le personnel infirmier qui y est associé.

\section{Un programme innovateur pour le personnel infirmier}

Les discussions menées dans notre centre de cancérologie sur la meilleure façon de relever le défi de soutenir les infirmières en oncologie, ont débouché sur la création d'un programme intitulé Prendre soin des soignants professionnels. L'initiative consistait en un séminaire de travail d'une journée pour le personnel infirmier, en dehors de la présence du personnel d'encadrement et de direction. La journée, qui était un jour de travail rémunéré, s'est déroulée dans l'environnement familial de Wellspring, un organisme de soutien aux malades du cancer situé à proximité du centre de cancérologie. Cette initiative était un partenariat entre le centre de cancérologie et Wellspring. L'élaboration du programme pour le personnel infirmier s'appuyait sur l'expérience des gens de Wellspring dans la coordination de groupes de soutien destinés aux malades du cancer.

La journée avait été organisée en fonction des objectifs suivants :

- aider les infirmières à trouver un équilibre entre vie professionnelle et vie privée, et

- aider les infirmières à faire face aux exigences continuelles de la pratique de leur spécialité.

L'ordre du jour de la journée est présenté dans le tableau 1. Le programme était basé sur les principes de la formation des adultes, et les séances mettaient en jeu une variété de méthodes d'enseignement et d'apprentissage. La journée elle-même s'équilibrait entre plusieurs activités.
1. Le contenu pédagogique s'intéressait au traumatisme et au deuil au deuxième degré ainsi qu'à leur impact émotionnel et physique sur les professionnels de la santé. Dans le prolongement du contenu pédagogique actuel sur les sujets, il était prévu que cette information aiderait les infirmières à reconnaître la réalité de ces phénomènes dans leur vie de pratique et la normalité d'en ressentir les effets. On essayait également de fournir des mots et des concepts aux infirmières pour leur permettre de parler de leurs expériences de la souffrance et du deuil de leurs patients.

2. Les infirmières ont eu l'occasion d'échanger des récits à propos de leurs expériences personnelles de la souffrance et du deuil et ce, dans un environnement favorable. Elles ont parlé de leur pratique et des types de situations qu'elles avaient affrontées avec les patients et leur famille. Les infirmières des services de soins aux hospitalisés et des services de soins externes ont discuté ensemble par petits groupes de six à huit participantes. Chaque groupe comptait un animateur-formateur qualifié pour mener de telles activités de groupe.

3. Le contenu pédagogique partagé concernait notamment l'importance de l'autosoin et les stratégies pour un autosoin personnel à la maison et sur le lieu de travail. Cette séance avait pour but d'élargir la base de connaissances des infirmières sur les stratégies spécifiques qu'elles pouvaient employer.

4. Des démonstrations de techniques de relaxation (par ex visualisation, relaxation de tout le corps, yoga, massage) ont été présentées avec possibilité pour les participantes de s'exercer aux différentes techniques et d'en éprouver les effets. Les participantes pouvaient apprendre la pratique d'une technique particulière ainsi que d'en ressentir les effets. Même si elles choisissaient de ne pas y recourir pour elles-mêmes, elles pourraient à l'avenir en parler à d'autres collègues ou aux patients.

Le même programme a été reconduit plusieurs fois, afin de permettre au plus grand nombre possible d'infirmières d'y participer. Une deuxième série a été proposée sur le même format, mais concentrée sur différents exercices de relaxation. Les animateurs de ce séminaire d'une journée avaient une expérience professionnelle dans les domaines favorisant la réflexion et une grande habitude de la coordination de groupes de soutien. Tous appartenaient au cercle des animateurs-formateurs ou des responsables de programme de Wellspring.

Le séminaire d'une journée avait été annoncé par des dépliants distribués dans les services. Les membres du personnel ont fait savoir à leurs gestionnaires à quel moment ils désiraient y participer. Chaque séminaire a accueilli entre 20 et 24 infirmières, parmi lesquelles du personnel de soins aux hospitalisés (médecine/radiothérapie et

Tableau 1. Notre Programme : Prendre soin des soignants professionnels

Ordre du jour du séminaire

\begin{tabular}{|l|l|l|}
\hline Objectifs & Version 1 & Version 2 \\
\hline $\begin{array}{l}\text { Connaissances (apprentissage) } \\
\text { (nouveau contenu) matin }\end{array}$ & $\begin{array}{l}\bullet \text { Traumatisme/deuil au deuxième degré } \\
\text { - Discussion en groupes sur les expériences } \\
\text { personnelles au travail } \\
\bullet \text { Stratégies d'autosoin }\end{array}$ & $\begin{array}{l}\bullet \text { Traumatisme/deuil vécu au deuxième degré } \\
\bullet \text { Rôle de la personnalité } \\
\bullet \text { Discussion en groupes sur les expériences } \\
\text { personnelles au travail } \\
\bullet \text { Valeur de la connaissance de soi } \\
\bullet \text { Stratégies d'autosoin }\end{array}$ \\
\hline $\begin{array}{l}\text { Compétences (pratiquer } \\
\text { des techniques d'autosoin) }\end{array}$ & $\begin{array}{l}\bullet \text { Visualisation et relaxation } \\
\text { Après-midi } \\
\text { Exercices du corps et de l'esprit }\end{array}$ & $\begin{array}{l}\bullet \text { Journalisation } \\
\text { Y Visualisation et relaxation } \\
\bullet \text { Exercices du corps et de l'esprit } \\
\bullet \text { Massage }\end{array}$ \\
\hline $\begin{array}{l}\text { Réseautage (opportunité } \\
\text { de rencontrer d'autres } \\
\text { infirmières en oncologie) }\end{array}$ & $\begin{array}{l}\bullet \text { Environnement familial } \\
\bullet \text { Repas préparés par traiteur } \\
\text { - Temps réservé pour les conversations informelles }\end{array}$ & $\begin{array}{l}\bullet \text { Environnement familial } \\
\text { Repas préparés par traiteur } \\
\bullet\end{array}$ \\
\hline
\end{tabular}


chirurgie) et du personnel des services de soins externes. Étant donné que le programme a duré deux ans, de nombreuses personnes y ont participé deux fois.

\section{Méthodes d'évaluation}

Deux approches ont été utilisées pour évaluer les résultats du programme. À la fin de la journée de séminaire, toutes les participantes ont été invitées à remplir un questionnaire d'évaluation, destiné à recueillir leurs impressions sur le programme et à fournir une estimation des résultats obtenus dans l'immédiat. Les items du questionnaire d'évaluation invitaient leur rétroaction sur tous les aspects des activités de la journée et de l'apprentissage relativement aux différents objectifs. Ces questionnaires ont été recueillis par des volontaires de Wellspring et résumés pour chaque journée. Les réactions ont donné lieu à de petits ajustements dans les séances suivantes du programme.

Pour étudier dans le détail les résultats à long terme, des entrevues en profondeur ont été réalisées un an après le programme auprès de 21 infirmières ayant participé au séminaire de travail. Une lettre d'invitation personnalisée, exposant les objectifs des entrevues et incitant à la participation, a été envoyée à chaque infirmière qui avait assisté au programme pour les soignants. Afin de déterminer l'intérêt suscité par le projet, une assistante de recherche prenait contact avec ces dernières par téléphone. Les entrevues avec les personnes ayant accepté de participer étaient conduites par cette assistante à un moment et en un lieu à la convenance de chacune des infirmières de chevet.

Le guide d'entrevue a été élaboré par les auteures pour cette évaluation dans un format semi-dirigé. Les questions ont rassemblé les témoignages des infirmières sur ce qui s'était passé durant cette journée, leur point de vue sur le séminaire, et toutes les différences observables après y avoir participé, concernant les activités d'autosoin, les interactions avec les collègues, ou les interactions avec les patients et leur famille. Les personnes interrogées ont également été invitées à faire des suggestions sur le programme des séminaires à venir.

Toutes les entrevues ont été enregistrées et retranscrites ensuite littéralement par l'assistante de recherche. Les informations permettant d'identifier les participants ont été supprimées des transcriptions avant que le texte ne soit vu par la chercheuse principale. Cette dernière étant également la responsable du service des soins infirmiers, elle n'a pas écouté les enregistrements, afin d'éviter qu'elle ne puisse reconnaître les voix des infirmières de chevet ayant pris part aux entrevues.

L'analyse des transcriptions s'est concentrée sur l'analyse du contenu et des thèmes (Silverman, 2000). La chercheuse principale a assuré la direction de cette étape du travail. Les transcriptions des entrevues ont été revues intégralement, et un code sommaire a été élaboré pour le contenu. Les entrevues ont ensuite été codées par l'un des membres de l'équipe (MF) selon les principes établis. Le code n'a pas subi de révision durant cette étape du processus. Le matériel codé a ensuite été revu et résumé pour chaque catégorie, de manière à refléter la gamme complète des idées exprimées, et des thèmes ont ainsi pu être dégagés.

\section{Résultats de l'évaluation}

\section{Partie I : Résultats quantitatifs}

Un total de 103 questionnaires d'évaluation a été retourné à la fin de huit séances d'une journée. La plupart des réponses provenaient d'infirmières qui travaillaient dans notre établissement depuis plus de cinq ans (voir le tableau 2).

Les personnes interrogées ont identifié l'apprentissage de techniques de relaxation comme le principal enseignement qu'elles attendaient de la séance. Quatre-vingts pour cent $(n=83)$ ont mentionné cet item comme la première raison ayant motivé leur participation. La deuxième attente le plus souvent citée était "d'apprendre sur le soutien émotionnel appliqué à soi-même » avec $36 \%(\mathrm{n}=37)$ des réponses des personnes interrogées reprenant cet item. Onze personnes seulement $(10,6 \%)$ ont évoqué le réseautage professionnel et la communication avec d'autres infirmières en oncologie comme le but de leur participation. Toutes, sans exception, ont indiqué que la séance avait répondu à leurs attentes.

Les participantes ont été conviées à dégager les principaux stresseurs de leur vie de pratique quotidienne, ou les types de facteurs qui influencent la manière dont elles ressentent leur rôle d'infirmière en oncologie et comment elles s'en accommodent. Un résumé de leurs réponses est présenté dans le tableau 3. Les descriptions démontrent clairement que les infirmières sont confrontées quotidiennement à un énorme stress et que des initiatives telles que le programme du séminaire sont indispensables pour les soutenir. Ces stress étaient souvent au centre des témoignages échangés par les infirmières dans les discussions de groupe tenues dans le cadre du séminaire.

Toutes les participantes ont jugé le séminaire utile (26\%) ou extrêmement utile (79 \%), et se sont déclarées satisfaites du programme; $98 \%$ ont fait savoir qu'elles le recommanderaient à leurs collègues. Le principal reproche fait à ce programme était qu'il était trop court. Quatre-vingt-cinq pour cent des personnes interrogées ont indiqué vouloir consacrer plus de temps à divers aspects du programme. Les types de commentaires écrits dans le questionnaire étaient les suivants :

- Merci. C'était formidable!

- Sensé, réaliste, agréable. On aimerait avoir ça régulièrement pour le personnel.

- Très riche en informations et très bénéfique.

- Ça m'a fait mieux prendre conscience. Ça m'a donné un aperçu très pointu.

- Ça m'a appris que tout le monde traversait les mêmes épreuves que moi - les mêmes expériences.

- Une très bonne occasion de me revigorer.

- C'était une journée paisible et relaxante, loin de tous les stress du travail.

Toutes les personnes interrogées ont déclaré qu'elles assisteraient de nouveau au programme. Quatre-vingt-un pour cent d'entre elles se sont dites prêtes à prendre sur leur temps libre pour y participer (c.-à-d. en dehors de leurs heures de travail), et plus de la moitié (59\%) ont indiqué qu'elles aimeraient participer à un programme comme celuici une fois par an. Les autres voulaient que le programme soit proposé plus souvent.

Enfin, les participantes ont formulé fort peu de suggestions pour améliorer le programme. De légères modifications du temps accordé à certains sujets et l'idée d'inclure le deuil parmi les sujets abordés ont été suggérées. La plupart des commentaires traduisaient le désir

\section{Tableau 2. Caractéristiques démographiques choisies des infirmières en oncologie participantes}

\begin{tabular}{|l|l|l|}
\hline Item & $\begin{array}{l}\text { Évaluation } \\
\text { quantitative }(\mathrm{N}=103)\end{array}$ & $\begin{array}{l}\text { Évaluation } \\
\text { qualitative }(\mathrm{N}=21)\end{array}$ \\
\hline $\begin{array}{l}\text { Personnel des soins } \\
\text { aux hospitalisés }\end{array}$ & 76 & 12 \\
\hline $\begin{array}{l}\text { Personnel des } \\
\text { soins externes }\end{array}$ & 27 & 9 \\
\hline $\begin{array}{l}\text { Ancienneté dans } \\
\text { l'établissement }\end{array}$ & 5 & - \\
\hline$<6$ mois & 12 & - \\
\hline $6-12$ mois & 11 & 2 \\
\hline $12,1-24$ mois & 10 & 3 \\
\hline 24,1 mois -5 ans & 65 & 16 \\
\hline$>5$ ans & 10 & - \\
\hline
\end{tabular}


de voir le programme proposé plus souvent, de donner à toutes les infirmières de chevet l'occasion d'y participer, d'ouvrir les séances à d'autres membres du personnel, et d'avoir des groupes de discussion semblables avec des gestionnaires.

\section{Partie II : Résultats qualitatifs}

Les entrevues avec les infirmières un an après leur participation au programme pour soignants professionnels ont duré de 20 à 60 minutes. Dans l'ensemble, les infirmières interrogées n'étaient pas capables de se remémorer les détails précis de l'information qui leur avait été présentée, mais elles avaient gardé un souvenir très net de la journée. Elles ont décrit leurs sentiments, les images que la journée faisait naître dans leur esprit, l'impression que leur avaient laissée les séances, les gens et leurs récits, ainsi que des échanges qui avaient eu lieu. Elles se sont rappelées avoir assimilé des messages ou idées clés sur le besoin d'équilibre dans leur vie, et avoir pris conscience que d'autres éprouvaient aussi les mêmes difficultés. En général, les infirmières ont expliqué comment elles s'étaient senties «bien soignées ». Le fait que l'établissement avait pris en charge financièrement leur participation à la séance en disait long sur l'importance que leur accordait leur employeur. Elles ont également décrit très clairement la nature des événements stressants de leur journée de travail (tableau 3).

La réalisation que leur établissement prenait soin d'elles et les aidait à gérer leur stress au travail s'est exprimé dans les déclarations suivantes :

Juste un jour sans travailler et on se détend. Et, en plus, on est payées. Ils sont larges avec l'argent... ils vous payent pour que vous preniez soin de vous-même. Et vous avez l'impression, vous avez

Tableau 3. Qu'est-ce qui est source de stress dans la vie quotidienne d'une infirmière en oncologie?

- Le manque d'équipement

- Le chaos régnant dans l'environnement de tous les jours

- Pas assez de personnel infirmier pour la somme de travail à accomplir

- Une lourde charge de travail/beaucoup de demandes

- Le manque de soutien et de compréhension de la part des gestionnaires et administrateurs.

- Ne pas se sentir appréciée à sa juste valeur

- Ne pas être écoutée par certains médecins

- Le manque de travail d'équipe

- Ne pas pouvoir évacuer ni montrer ses émotions sans crainte au travail

- Les patients sont très malades

- tre chaque jour confrontée à la mort et au mourir

- Voir en permanence la maladie et la souffrance

- Voir des gens plus jeunes tellement malades

- Perdre des gens dont on s'est occupé de nombreuses fois

- Voir le désespoir, la tristesse, le traumatisme humain, les émotions

- Les familles peuvent se montrer tellement exigeantes

- Des attentes peu réalistes des patients et des familles

- Les patients et leurs proches ne s'entendent pas sur ce qui devrait être fait

- Beaucoup de traitements agressifs et le refus d'admettre la proximité de la mort

- Jamais assez de temps pour faire ce qui devrait être fait

- Pas le temps de parler avec les patients

- Les médecins ne s'adressent pas honnêtement aux patients et aux familles

- Le travail déborde sur la vie privée

- Peu de temps ou d'occasion de résolution du deuil

- Ne pas être capable d'offrir aux patients ou aux proches des soins de qualité l'impression d'être quelqu'un. Vous savez, vous n'êtes pas seulement une soignante... il y a quelqu'un qui prend soin de vous, la soignante. Et ils vous donnent cette journée pour que vous quittiez votre poste de travail et preniez soin de vous. C'était excellent. (1)

J'avais juste l'impression que j'étais... qu'ils faisaient quelque chose pour nous dorloter un petit peu. Donc ils nous rendaient quelque chose, au lieu de prendre et de nous épuiser en permanence. (2)

\section{Bénéfices de la journée de séminaire proprement dite}

Quatre thèmes d'ensemble se sont dégagés des données des entrevues sur le programme du séminaire. Le premier thème soulignait l'idée que tout le monde avait eu du bon temps. En repensant à cette journée, les personnes interrogées se rappelaient un sentiment d'avoir été soignées et dorlotées. L'environnement familial de Wellspring, la nourriture, les exercices de relaxation, et l'idée que tout cela se déroulait pendant un jour de «travail » corroboraient ces impressions.

C'était comme un jour de récréation, presque... amusant, relaxant, des repas gratuits, une sieste, des discussions - et nous adorons toutes parler - et faire de l'exercice. C'était vraiment une bonne journée. Ça semblait comme, "Oh c'est fini? Oh, est-ce qu'il faut déjà partir? », vous savez. Et d'habitude, on est toujours pressées de partir d'ici, pas vrai? Alors je pense que c'était vraiment quelque chose de positif. Je pense qu'ils ont trouvé quelque chose de bien ici... ils ne nous ont pas du tout donné l'impression que c'était une journée de formation.(3)

Le deuxième thème se rapportait à la valeur de la relation. Les infirmières ont évoqué l'occasion qui leur avait été fournie de parler avec d'autres infirmières en oncologie de ce qui leur arrivait dans leur pratique quotidienne et de leurs réactions personnelles à ces situations. Elles ont reconnu des similitudes dans les événements et dans leurs réactions, dont elles n'avaient pas nécessairement conscience avant de participer à ce séminaire. La présence hétérogène de personnel des services de soins aux hospitalisés et des services de soins externes a renforcé le sentiment de surprise qui caractérisait la réalisation qu'elles éprouvaient toutes des stress similaires. Cela a permis de développer, chez elles, l'idée qu'elles formaient une équipe d'infirmières au sein d'un programme de lutte contre le cancer.

...c'était une expérience de relation plus qu'une expérience d'apprentissage. Juste être capables de s'écouter les unes les autres parler. Nous vivons toutes tant d'expériences différentes. (8)

...Ce qui était bien, c'était que tout le monde ressentait le même genre de choses. Vous savez, comme quand vous êtes persuadée qu'il n'y a que vous qui sentez les choses d'une certaine manière, mais en fait, c'est la plupart d'entre nous. (3)

La notion d'être ensemble et de parler les unes avec les autres des événements et des réactions émotionnelles - était vue comme quelque chose d'improbable dans le déroulement ordinaire d'une journée, à plus forte raison entre infirmières de différents services. D'être capables de participer aux discussions dans l'atmosphère du séminaire a permis aux infirmières de dépasser le simple fait de se plaindre des stress qu'elles éprouvent. Elles ont réussi à parler en toute confiance de leurs réactions émotionnelles, parfois très personnelles. Le fait de réaliser que d'autres avaient des réactions et des émotions semblables a permis d'installer une relation. Soudainement, les infirmières avaient le sentiment de ne pas être seules face aux situations de leur vie de pratique quotidienne.

Vous savez, parfois vous pensez que vous êtes complètement isolée. C'est le côté qui m'a vraiment touchée. Juste de pouvoir partager l'impact émotionnel de notre travail. Et je pense que rouspéter sur des choses qui nous dérangent, vous savez... on n'en a pas l'occasion [ici 
dans le service]. Mais de sortir de l'environnement de travail et de parler avec les infirmières d'autres services, vous réalisez qu'il n'y a pas que vous qui ressentez ça. (19)

Le troisième thème décrivait le bénéfice que les infirmières pensaient avoir retiré du séminaire, la valeur du soutien. L'opportunité d'être ensemble et de partager les unes avec les autres a fait naître un sentiment de soutien mutuel. Les discussions ont fourni aux infirmières des occasions de faire entre elles des commentaires, du genre «tu as bien géré ça », «j'aurais été bouleversée, moi aussi », " rien d'étonnant à ce que tu te sentes frustrée ». Ces types de commentaires étaient considérés comme traduisant de la compréhension et de l'approbation. Tout à coup, des comportements observés chez les collègues avaient davantage de sens. Elles ont commencé à mieux s'expliquer pourquoi les gens agissaient comme ils le faisaient ou disaient ce qu'ils disaient dans certaines situations. Elles ont commencé à mieux saisir les défis auxquels elles étaient toutes confrontées et à mieux comprendre comment les stress et les tensions pouvaient entraîner certaines réactions et certains comportements. Et elles ont pu commencer à exprimer cet entendement.

C'était l'occasion d'exprimer ce qu'on endurait. Et, certainement, vous savez, les gens en parlaient... et vous appreniez à connaître les autres... et vous pouvez désormais être plus compréhensive envers celles avec qui vous travaillez... c'était comme si nous étions devenues une équipe soudée... Et la manière dont on discutait de nos inquiétudes, c'était vraiment comme une thérapie de groupe. (4)

Nous avons eu de bons échanges... du bon temps et de bons échanges, ainsi nous savons que nous ne sommes pas seules... Nous savons... nous sommes toutes très stressées. Et c'est vraiment bien, cette réalisation. Car nous savons maintenant que nous ne sommes pas particulièrement stressées, mais que c'est en quelque sorte la norme. Et je pense que ça nous aide beaucoup. (9)

Le quatrième thème dégagé de ces entrevues était la valeur des récits. Faire des récits dans les séances de groupe était un outil efficace de mise en relation et de soutien. Au moyen de récits, les infirmières se confiaient et échangeaient leurs points de vue. La méthode leur a permis de trouver un terrain commun. Le fait de faire des récits est en soi une expérience humaine commune à tous les êtres, un exercice dans lequel beaucoup se sentent à l'aise. Ce contexte de partage de récits relatifs au travail avec les collègues a permis de concentrer l'attention des participantes et de faciliter l'apprentissage.

Les interactions avec les autres infirmières. Découvrir leur personnalité et comment elles se perçoivent en tant qu'infirmières. Et simplement écouter leurs récits... nous nous apprécions davantage les unes les autres... elles ont partagé les relations qu'elles entretiennent avec les patients et leur famille. Simplement, ce qu'elles ont dit m'a fait beaucoup de bien, parce qu'elles jetaient un regard perspicace sur leurs patients et sur elles-mêmes. (20)

Je pense que quand nous avons partagé des récits... les choses qui nous sont arrivées, des histoires personnelles, cela nous a permis de sortir tout ça de notre système. C'était un aspect important. Quand nous partageons comme ça, nous construisons une relation avec les autres personnes présentes... Ça revient à jeter des ponts entre nous. (5)

\section{Bénéfices après la journée de séminaire}

On a demandé aux participantes de décrire les enseignements ou autres bénéfices significatifs qu'elles estimaient avoir retirés du séminaire et de dire si ces bénéfices avaient continué à se faire sentir dans le temps. Elles ont fait état de bénéfices pour elles-mêmes et leur famille, dans leur vie professionnelle, et dans les soins dispensés aux patients et à leur famille.
Les principaux bénéfices décrits par les infirmières étaient les bénéfices pour elles-mêmes. Elles avaient le sentiment d'avoir appris beaucoup de choses à cette séance, même si, au moment de l'entrevue, les détails étaient plutôt flous. L'enseignement le plus profitable sur le long terme avait été celui des nouvelles techniques de relaxation et de nouveaux aperçus pointus. Elles avaient découvert de nouveaux exercices de relaxation durant le séminaire et avaient pu en faire usage par la suite.

Pour moi, personnellement, les techniques de relaxation, je pense. De les utiliser pour m'aider à faire face, des choses comme ça... d'être plus consciente d'une situation de stress... de réaliser quand ça vous arrive... quand ça vous affecte et que vous pouvez y changer quelque chose. (15)

Eh bien, je respire profondément et tout ça, chaque fois que je peux. Toujours quand je rentre chez moi. Je respire un bon coup. Ouais. Et il y a des fois ici, en fait, où vous avez eu une journée tellement dure qu'il vous semble que vous devez prendre du recul, pour évacuer les choses, avant d'exploser ou de faire quelque chose de stupide. Donc il y a des fois où il faut vraiment s'en éloigner. Il faut vraiment le faire. (13)

L'information sur le stress et sur les stratégies d'adaptation a également amené les infirmières à prendre mieux conscience des situations qui engendrent du stress, des raisons pour lesquelles elles sont stressantes et des options qui permettent d'y faire face.

Pour ma part, j'ai appris à m'adapter, c'est ça. À mieux m'adapter et surtout à mieux surmonter la mort et le mourir. J'avais du mal avec ça. (14)

Je dirais que maintenant, je prends une décision consciente quand je choisis d'être affectée par une situation... les politiques et tout ce qui s'en suit. J'essaye peut-être d'éviter ce genre de stress. (15)

Les infirmières ont aussi gagné une meilleure compréhension et appréciation de l'impact du deuil et du chagrin et de l'influence qu'ils peuvent avoir sur le comportement. Cet aperçu pointu les a aidées à avoir plus de patience et une plus grande capacité à voir et à comprendre leurs expériences et celles des autres. Ainsi, elles se sont senties plus ouvertes, plus à l'écoute des autres, et prêtes à reconnaître la réalité de leur stress au travail.

C'est permis d'être humain, d'avoir du chagrin, presque. Et si vous avez une mauvaise journée, une mauvaise expérience, vous avez. le droit de ressentir cette émotion et même de l'exprimer... Je suis plus consciente de ça qu'avant. Spécialement en écoutant les collègues...je suis plus réceptive envers elles. (16)

Une chose que ça m'a apportée, ça a normalisé mes émotions. Je sais que je ne suis pas seule... Je suis occupée et stressée par moments, mais c'est bon d'entendre les autres dire cela et communiquer le même genre d'émotions que les vôtres... vous réalisez que pour les gens qui travaillent en oncologie, c'est la norme... et c'est bien de réaliser ça. (9)

Quelques-unes de mes collègues étaient présentes ce jour-là, et certains récits, bon, c'est ce que j'ai réalisé, m'ont apporté une perspective différente, un respect, une sympathie, simplement une compréhension que, après tout, ma vie n'est pas la seule qui n'a pas été facile. Alors peut-être que ça m'a donné un peu plus de compassion. (11)

La notion de prendre du temps pour soi était également citée comme une perspective importante, encore présente aujourd'hui chez les participantes. Le séminaire a démontré à quel point il était important de prendre ce temps. Lorsqu'on n'a pas de temps pour soi, l'équilibre dans la vie personnelle et la capacité de gérer les stress de 
la vie professionnelle s'en trouvent diminués. Par conséquent, le séminaire est devenu pour certaines infirmières une incitation à mettre davantage l'accent sur leurs activités personnelles.

C'était bon de savoir que mes émotions sont normales... donc à court terme, il y avait confirmation... à long terme, bon, je suppose le renforcement des capacités à s'occuper de soi-même, des techniques de relaxation, des habiletés fondamentales. Je suppose qu'en les renforçant, vous les intégrez... et qu'il est important de prendre soin de soi-même avant de prendre soin des autres. (18)

Comment être bon avec soi-même... bien se reposer, bien dormir, faire de l'exercice... avoir une sorte de vie sociale normale en dehors $d u$ travail pour pouvoir laisser le stress derrière... et la séance m'a aidée à prendre conscience que si je voulais ressentir un sentiment de bien-être, être détendue alors que le travail est plus stressant... il y a des choses que je pouvais faire... (17)

Une partie des changements entrepris par les infirmières a été de consacrer davantage de temps à leur famille. Elles ont eu le sentiment qu'elles n'avaient pas été les seules à en profiter et que leur famille avait aussi bénéficié de cette évolution.

Ça m'a vraiment fait penser à ma famille et au temps que je passais avec mes filles. Elles ont besoin de moi, elles aussi. Je ne peux pas laisser le travail me retirer ça. (7)

En terme de vie professionnelle, les infirmières ont jugé que le séminaire avait eu un impact à long terme sur leur manière d'apprécier leurs collègues et leur capacité à se soutenir les unes les autres. Leur compréhension rehaussée des expériences des unes et des autres et la reconnaissance qu'elles passaient toutes par des moments difficiles signifiaient qu'elles pouvaient être plus tolérantes. Elles ont éprouvé un sentiment partagé de travailler ensemble, ainsi qu'un réconfort à l'idée que les autres étaient sur la même longueur d'ondes.

Quelquefois, dans tous les environnements comme celui-ci, les gens sont accablés à différents degrés... ils en arrivent à un point où ils maintiennent tout juste la tête hors de l'eau. Ils n'ont pas l'énergie d'en faire plus. Leur énergie va aux patients et aux familles. Il en reste très peu pour les autres...ce genre de séance nous aide à réaliser cela. (17)

C'est réconfortant de savoir qu'il y a des infirmières partout dans l'hôpital, en oncologie, qui sont confrontées aux mêmes situations. Des expériences semblables, et elles en sont affectées différemment mais pareillement. Je ne sais pas, c'est bon de savoir ça. (15)

J'ai l'impression que je ne suis pas la seule à ressentir ce que je ressens par rapport aux soins que je donne aux patients atteints du cancer. Et aussi que je sais que je ne suis pas seule. Il y a d'autres gens qui ressentent les mêmes choses que moi. (14)

En ce qui concerne les patients et les proches, les infirmières ont rapporté qu'elles manifestaient davantage d'empathie et de compréhension envers les situations de ces derniers. Elles ont appliqué à leurs patients, certains aspects de ce qu'elles avaient appris sur le deuil et le chagrin. Cela leur a permis d'être plus patientes, de ralentir le rythme durant leurs interactions avec les gens, et de ne pas avoir peur d'aborder les sujets émotionnels. Elles ont réalisé qu'elles étaient mieux préparées à demander aux patients ce qui pouvait bien les tracasser et à prendre le temps d'écouter leurs réponses.

Avec les patients, les familles, bon, j'écoute probablement plus attentivement. Je suis probablement mieux accordée et probablement je me concentre plus sur leurs expériences à ce moment-là, dans une situation difficile. (16)

Je pense que je parle plus avec les patients. Et de les amener à exprimer davantage leurs sentiments et leur état d'esprit, et de chercher ce qu'ils ressentent, au lieu de faire juste le boulot technique... ça crée une meilleure relation avec mes patients et une relation plus confiante. Je sens que je peux faire plus pour eux une fois que j'arrive à mieux les connaître et à savoir quels sont leurs besoins. (10)

Finalement, un sentiment définitif de ne plus être seule, de ne pas avoir à tout porter sur ses seules épaules, est apparu. Au milieu de l'animation et du stress d'un jour de travail, il est si souvent facile de ne voir uniquement ce qui doit être fait et ce que l'on doit faire individuellement; il est facile d'oublier qu'il y a les autres autour.

C'était plus un sentiment de compréhension et de camaraderie avec toutes celles qui étaient là...J'en suis partie en me sentant bien à propos du travail que nous faisions...j’y ai gagné en fierté... nous faisons vraiment quelque chose d'utile... Je pense que j'ai un plus grand respect pour les personnes que sont les infirmières qui travaillent ici. (13)

En tant qu'infirmière, vous ne dispensez pas des soins infirmiers en oncologie toute seule. Vos collègues ressentent les choses de la même manière que vous... quelquefois, vous vous sentez vraiment désemparée... d'autres fois, vous demandez pourquoi? Pourquoi estce que cette personne est clouée avec le cancer? (14)

\section{Discussion}

Le programme, Prendre soin des soignants professionnels, a été élaboré pour fournir aux infirmières de chevet une opportunité d'apprendre davantage sur les stratégies d'adaptation aux situations de stress auxquelles elles sont confrontées dans leur pratique quotidienne en oncologie. Il a été conçu pour délivrer une nouvelle information, augmenter les compétences et élargir les réseaux existants entre les infirmières en oncologie.

Plus qu'une surprise, le succès du programme a dépassé les espérances. Les résultats quantitatifs et qualitatifs fournissent un aperçu précieux sur les raisons de ce succès. Ils révèlent également ce qui est utile pour soutenir les infirmières en oncologie, qui travaillent dans les environnements de pratique stressants d'aujourd'hui.

Le programme a été élaboré selon les concepts de la formation des adultes. Plusieurs principes sont apparus comme les clés de la réussite du programme : les sujets étaient ceux que les apprenantes trouvaient les plus importants et les plus pertinents quant à elles, les apprenantes avaient une expérience et une compétence reconnues relativement à ces sujets, et les méthodes d'enseignement étaient hautement interactives et offraient la possibilité d'une mise en pratique immédiate. Ces infirmières connaissaient parfaitement le stress quotidien que représentent les soins infirmiers en oncologie et mesuraient toute l'importance des stratégies d'adaptation. Les discussions de groupe ont fourni de nombreuses occasions de partager des récits, de parler aux autres de sa vie de pratique, et d'apprendre chacune des témoignages des autres. L'apprentissage des techniques de relaxation était amusant, pratique et motivant. Il faut noter cependant qu'en la matière, les responsables et les animateurs étaient parfaitement préparés pour cette approche pédagogique et ce travail de groupe. Le leadership est un élément clé de la conduite des séances et de l'atteinte des buts fixés.

La valeur de la relation avec ses pairs et du soutien qu'apporte le partage de récits relatant des expériences semblables a été citée par d'autres chercheurs. En particulier, des bénéfices comparables ont été évoqués par des patients atteints du cancer relativement aux groupes d'entraide (Gray, Fitch, Davis et Phillips, 1997a; Gray, Fitch, Davis et Phillips, 1997b). Un fort sentiment de solidarité se fait jour lorsque les gens réalisent qu'ils ont en commun des expériences et des émotions, et qu'ils ont traversé les mêmes situations. Le sentiment d'isolement et de solitude s'amenuise. Se décrire les unes aux autres ces situations, faire le récit de ce qui s'est passé, constituent un moyen de trouver et de partager des points communs. Les récits comportent des idées sur les façons de gérer le stress ou de maîtriser les situations, 
ainsi que des illustrations pouvant servir à normaliser et à valider les émotions ou les réactions. Lorsqu'un animateur sait bien utiliser cet outil pour le travail en groupe, cette approche peut être très efficace.

Les résultats de cette évaluation ont des implications pour les gestionnaires et les administrateurs relativement aux stratégies qui favorisent la création de postes de travail de qualité pour les infirmières en oncologie. Les infirmières de chevet ont souligné l'importance du soutien de gestionnaires conscients de la pression qu'elles subissent dans le travail (Bakker et al., sous presse). Offrir ce programme et faire en sorte qu'il soit rémunéré comme un jour travaillé a été clairement perçu comme un soutien et une reconnaissance de la part de l'établissement. La nature du programme, ainsi que le cadre choisi pour le dispenser et la nourriture servie, ont donné aux infirmières le sentiment d'être dorlotées. Cela a été interprété comme une marque supplémentaire de l'intérêt que l'établissement porte au bien-être du personnel infirmier. Le contenu du programme et son ordre du jour ont ouvert la voie à de nouvelles façons pour les participantes de prendre soin les unes des autres. Le respect mutuel s'est fortifié à mesure qu'elles parlaient et s'enrichissaient de leurs récits respectifs. Cette sollicitude a alimenté le sentiment d'appartenance à une équipe et l'impression de ne pas être seule face à l'adversité. On sent qu'on appartient à la communauté des infirmières en oncologie, lesquelles font la différence pour les gens qui vivent avec le cancer. Le programme a fourni aux participantes des aperçus sur la nature du stress au travail et sur les façons de mieux gérer ou maîtriser la réalité qu'elles doivent affronter tous les jours.

Cette étude comporte des limites. Étant donné que le programme s'adressait aux infirmières d'un seul programme de lutte contre le cancer, les données recueillies proviennent uniquement des

\section{Références}

Bakker, D., Fitch, M.I., Green, E., Butler, L., \& Olson, K. (sous presse). Les soins infirmiers en oncologie : un système de soins en évolution

Cohen, M.Z., \& Sarter, B. (1992). Love and work: Oncology nurses' views of the meaning of their work. Oncology Nursing Forum, 19(10), 1481-6.

Cohen, M.Z., Haberman, M.R., Steeves, R., \& Deatrick, J.A. (1994). Rewards and difficulties of oncology nursing. Oncology Nursing Forum, 21(8, suppl.), 9-17.

Corner, J. (2002). Nurses experiences with cancer. European Journal of Cancer Care, 11, 193-199.

Feldstein, M.A. \& Gemma, P.B. (1995). Oncology nurses and chronic compounded grief. Cancer Nursing, 18(3), 228-236.

Fitch, M., \& Steele, R. (2003). Supportive care needs of cancer patients: Looking beyond the obvious. Supportive Care in Cancer, 11(6), 423.

Gray, R., Fitch, M., Davis, C., \& Phillips, C. (1997a). Interviews with men with prostate cancer about their self-help group experiences. Journal of Palliative Care, 13(1), 15-21.

Gray, R., Fitch, M., Davis, C., \& Phillips, C. (1997b). A qualitative study of breast cancer self-help groups. Psycho-oncology, 6, 279289.

King, P., \& Jordan-Welch, M. (2003). Nurse-assisted suicide: Not an answer in end-of-life care. Issues on Mental Health Nursing, 24(1), 45-57.

Kushnir, T., Rabin, S., \& Azulai, S. (1997). A discipline study of stress management in a group of pediatric oncology nurses. Cancer Nursing, 10(6), 414-421.

Lamkin, L., Rosiak, J., Buerhaus, P., Mallory, G., \& Williams, M. (2002). Oncology Nursing Society workforce survey. Part II: Perceptions of the nursing workforce environment and adequacy of nurse staffing in outpatient and inpatient oncology settings. Oncology Nursing Forum, 29(1), 93-100. infirmières travaillant dans ce centre. En outre, la majorité des infirmières qui ont participé à l'évaluation oeuvraient en oncologie depuis plus de cinq ans. Il s'agit d'une réflexion réaliste émanant du personnel d'un centre de cancérologie, mais les opinions peuvent ne pas refléter la perspective de celles qui sont nouvelles dans le monde de la lutte contre le cancer. Ce type de programme peut ne pas être aussi efficace auprès des infirmières qui débutent en oncologie.

\section{Implications}

Sans aucun doute, ce programme, qui propose de prendre soin des soignants professionnels, servait un but utile. À la lumière de l'avenir qui se dessine pour les soins en cancérologie et des pressions continues dans cet environnement de travail, un programme tel que celui qui est décrit ici devrait être proposé à intervalles réguliers. Les soins infirmiers en oncologie sont une spécialité qui cause beaucoup de stress, et aider les infirmières de chevet à gérer ce stress revêt une grande importance. Les bénéfices de notre programme ont été perçus tant au niveau personnel qu'au niveau de la prestation des soins. À l'avenir, de nouvelles initiatives de recherche pourraient déterminer si ces observations se confirment lorsqu'un tel programme est offert dans d'autres programmes ou contextes de lutte contre le cancer. Les liens entre la participation à un programme de ce type et la diminution des sentiments de stress ou la baisse des taux d'absentéisme, pourraient également être explorés. Finalement, la formation de partenariats avec des organismes compétents dans l'organisation de tels programmes peut constituer une approche intéressante pour les responsables des programmes de lutte contre le cancer, soucieux de procurer une assistance à leur personnel.
Lancely, A. (2001). The impact of cancer on health professionals. In T. Corner \& C. Barley (Eds.), Cancer nursing - Care in context. Oxford, Blackwell Science.

Larson, D. (1992). The challenge of caring in oncology nursing. Oncology Nursing Forum, 19, 857-861.

Lev, E.L. (1994). Issues for the nurse caring for the dying patient. Oncology Nursing: Patient Treatment and Support, 1(1), 1-10.

Lewis, A.E. (1999). Reducing burnout: Development of an oncology staff bereavement program. Oncology Nursing Forum, 26(6), 1065-1069.

Loney, M. (1998). Death, dying and grief in the face of cancer. In C. Burke (Ed.), Psychosocial dimensions of oncology nursing care. Pittsburg: Oncology Nursing Press Inc.

Lovern, E. (2001). This shortage needs CPR: Studies at odds on whether there are enough to go around. Modern Healthcare, 31(24), 4-5, 16.

McElroy, A.M. (1982). Burnout: A review of the literature with application to cancer nursing. Cancer Nursing, 5(3), 211-217.

Newlin, N.J., \& Wellisch, D.K. (1978). The oncology nurse: Life on an emotional roller coaster. Cancer Nursing, 1(6), 447-449.

Saunders, J.M., \& Valente, S.M. (1994). Nurses' grief. Cancer Nursing, 17(4), 318-325.

Silverman, D. (2000). Doing qualitative research: A practical handbook. London: Sage.

Vachon, M.L.S. (1986). Myths and realities in palliative/hospice care (pp. 63-79). Hawthorne, NJ: Hawthorne Press.

Wilkinson, S.M. (1994). Stress in cancer nursing - does it really exist? Journal of Advanced Nursing, 20, 1079-1084.

Yasko, J.M. (1983). Variables which predict burnout experienced by oncology nurse specialists. Cancer Nursing, 6(2), 109-116. 